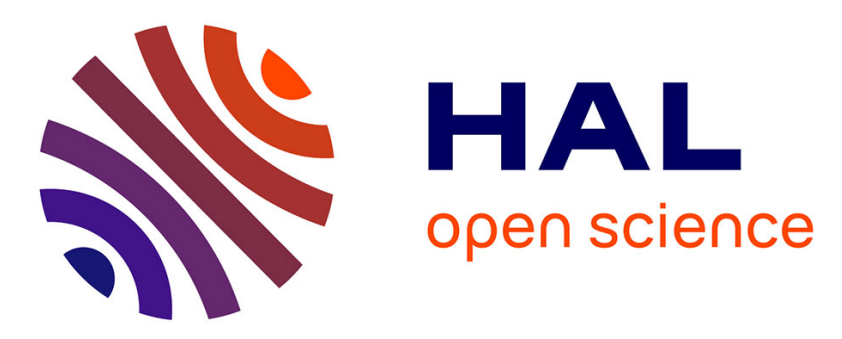

\title{
Far-ultraviolet aurora identified at comet 67P/Churyumov-Gerasimenko
}

M. Galand, P. D. Feldman, D. Bockelée-Morvan, N. Biver, Y.-C. Cheng, G. Rinaldi, M. Rubin, K. Altwegg, Jan Deca, A. Beth, et al.

\section{- To cite this version:}

M. Galand, P. D. Feldman, D. Bockelée-Morvan, N. Biver, Y.-C. Cheng, et al.. Far-ultraviolet aurora identified at comet 67P/Churyumov-Gerasimenko. Nature Astronomy, 2020, 4, pp.1084-1091. 10.1038/s41550-020-1171-7 . hal-03021646

\section{HAL Id: hal-03021646 \\ https://hal.science/hal-03021646}

Submitted on 19 Nov 2021

HAL is a multi-disciplinary open access archive for the deposit and dissemination of scientific research documents, whether they are published or not. The documents may come from teaching and research institutions in France or abroad, or from public or private research centers.
L'archive ouverte pluridisciplinaire HAL, est destinée au dépôt et à la diffusion de documents scientifiques de niveau recherche, publiés ou non, émanant des établissements d'enseignement et de recherche français ou étrangers, des laboratoires publics ou privés. 


\section{Far ultraviolet aurora identified at comet 67P/Churyumov- Gerasimenko}

3 $\quad$ M. Galand ${ }^{1}$, P. D. Feldman ${ }^{2}$, D. Bockelée-Morvan ${ }^{3}$, N. Biver ${ }^{3}$, Y.-C. Cheng ${ }^{3}$, G. Rinaldi ${ }^{4}$, M. Rubin ${ }^{5}$,

4 K. Altwegg ${ }^{5}$, J. Deca ${ }^{6,7}$, A. Beth ${ }^{1}$, P. Stephenson ${ }^{1}$, K. L. Heritier ${ }^{1}$, P. Henri ${ }^{8}$, J. Wm. Parker ${ }^{9}$,

5 C. Carr $^{1}$, A. I. Eriksson ${ }^{10}, \&$ J. Burch ${ }^{11}$

$6{ }^{1}$ Department of Physics, Imperial College London, Prince Consort Road, London, SW7 2AZ, UK

$7 \quad{ }^{2}$ Department of Physics and Astronomy, The Johns Hopkins University, 3400 N. Charles Street,

8 Baltimore, MD 21218, USA

$9{ }^{3}$ LESIA, Observatoire de Paris, Université PSL, CNRS, Sorbonne Université, Université de Paris,

5 place Jules Janssen, 92195 Meudon, France

${ }^{4}$ IAPS-INAF, via del Fosso del Cavaliere, 100, 00133 Roma, Italy

${ }^{5}$ Physikalisches Institut, University of Bern, Sidlerstrasse 5, 3012 Bern, Switzerland

${ }^{6}$ Laboratory for Atmospheric and Space Physics (LASP), University of Colorado Boulder, Boulder, CO 80303, USA

${ }^{7}$ Institute for Modeling Plasma, Atmospheres and Cosmic Dust, NASA/SSERVI, Moffet Field, CA 94035, USA

${ }^{8}$ LPC2E, CNRS, Université d'Orléans, 45071 Orléans, France

${ }^{9}$ Southwest Research Institute, Department of Space Studies, Suite 300, 1050 Walnut Street, Boulder, CO 80302, USA

${ }^{10}$ Swedish Institute of Space Physics, Ångström Laboratory, Lägerhyddsvägen 1, 75237 Uppsala, Sweden 
${ }^{11}$ Southwest Research Institute, P.O. Drawer 28510, San Antonio, TX 78228-0510, USA

Having a nucleus darker than charcoal, comets are usually detected from Earth through the emissions from their coma. The coma is an envelope of gas which forms through the sublimation of ices from the nucleus, as the comet gets closer to the Sun. In the far ultraviolet, observations of comae have revealed the presence of atomic hydrogen and oxygen emissions. When observed over large spatial scales as seen from Earth, such emissions are dominated by resonance fluorescence pumped by solar radiation. Here we analyse data obtained close to the cometary nucleus by the Rosetta spacecraft. In order to identify their origin, we undertake a quantitative multi-instrument analysis of the far ultraviolet emissions by combining coincident neutral gas, electron, and spectroscopic observations together. We establish that the atomic emissions detected from Rosetta around comet 67P/Churyumov-Gerasimenko at large heliocentric distances result from the dissociative excitation of cometary molecules by accelerated solar-wind electrons (and not electrons produced from photo-ionisation of cometary molecules as suggested in past studies). We reveal their auroral nature. Similarly to the discrete aurorae at Earth and Mars, this newly-discovered cometary aurora is driven by the interaction of the solar wind with the local environment. We highlight how OI $1356 \AA$ could be used as a tracer of solar-wind electron variability.

The Rosetta spacecraft escorted comet 67P/Churyumov-Gerasimenko (referred as 67P hereafter) for more than two years ${ }^{1,2}$. Onboard, the Alice ultraviolet imaging spectrograph ${ }^{3}$ detected Far UltraViolet (FUV) atomic hydrogen and oxygen emissions ${ }^{4-7}$ from the cometary coma. Spec- 
troscopic analysis of these emissions shows that their origin seems to be consistent with the dissociative excitation of cometary molecules, such as $\mathrm{H}_{2} \mathrm{O}$ and $\mathrm{O}_{2}{ }^{8}$, by electrons ${ }^{4,7}$. The same process is taking place at the Jovian moons, Ganymede ${ }^{9,10}$ and Europa ${ }^{11}$, though the magnetic and particle environments are very different. Observed from Earth, the FUV atomic emissions from comets primarily result from the resonance fluorescence ${ }^{12}$ pumped by solar radiation and occurring in atoms in the extended coma. These atoms are produced by photodissociation of cometary molecules by solar radiation. The electrons thought to be responsible for the excitation of FUV emissions observed from Rosetta are supposed to be photoelectrons resulting from the ionisation of cometary neutrals by solar Extreme UltraViolet (EUV) radiation ${ }^{4,7}$. This means that the FUV emissions are presumed to be dayglow which primarily results from the interaction of solar photons with an atmosphere or a coma. In contrast, auroral emissions - as defined here - originate from the interaction of energetic, extra-atmospheric particles with an atmosphere or, more generally, the envelope of gas surrounding a planetary body ${ }^{13}$. By "energetic", we refer to particles energetic enough to trigger the excitation which leads to emission. The energy range varies with the auroral process. For dissociative excitation of water, the minimum energy required for the FUV lines analysed here are between 14 and $17 \mathrm{eV}$. The planetary body does not need to have an intrinsic magnetic field to host aurorae. However, to be auroral, emissions need to be driven by energetic particles whose source is external (that is, not locally produced, like photoelectrons).

Northern and southern lights, the so-called aurora illuminating the high latitude skies on Earth, have captured the human imagination for centuries. They are highly relevant for providing a snapshot of the particle energy input over the high latitude regions and play a key role in space 
weather. Over the past half century, auroral emissions have been discovered at planets and moons in the Solar System ${ }^{13-15}$ and beyond ${ }^{16}$. Aurora is a universal phenomenon, accessible to observations and analysis: aurora is a tracer of plasma interaction, a remote-sensing of magnetic field configuration, and a fingerprint of particle sources and atmospheric species ${ }^{13}$. So far, at comets, auroral emissions have been reported in the X-rays and EUV, resulting from the interaction of heavy solar-wind ions with cometary gases ${ }^{13,17}$. Here we undertake a multi-instrument analysis of FUV atomic emissions (HI Ly $\beta$ line and OI $1356 \AA$, and OI $1304 \AA$ multiplets), by combining coincident Rosetta datasets together and comparing observed and modelled brightnesses. Observations of the energetic (10-200 eV) electron distribution, neutral gas (in situ and remote), and FUV emissions, acquired over similar time periods at large heliocentric distances ( $\geq 2 \mathrm{AU})$, are linked together through a physics-based model (Fig. 1). We apply this approach to nadir- and limb-viewing configurations in order to underpin the mechanism producing the FUV atomic emissions, to identify the origin of the energetic source and to reveal the nature of the emissions.

In order to establish the source of the FUV atomic emissions in a quantitative manner, the multi-instrument analysis is applied to seven nadir-viewing cases (see Table 1). The selected cases correspond to viewing over the shadowed nucleus: this avoids any contamination of the FUV emissions by solar radiation reflected off the nucleus' surface ${ }^{6}$. We are only focusing on $\mathrm{HI}$ and OI emissions here: the selected cases are for viewing over the northern hemisphere where water is the dominant species in the coma during the periods of interest ${ }^{18,19}$.

Comparing observed (magenta) and modelled (black) FUV brightnesses for the five 2015- 
2016 nadir-viewing cases shows that the HI and OI emissions are produced by the dissociative excitation of cometary neutrals by energetic electrons (Fig. 2). The composition $\left(\mathrm{H}_{2} \mathrm{O}, \mathrm{CO}_{2}, \mathrm{CO}\right.$, and $\mathrm{O}_{2}$ ) and total column density of the neutral gas are obtained from in situ observations from the Rosetta Orbiter Spectrometer for Ion and Neutral Analysis (ROSINA) ${ }^{20}$. The emission frequency is derived from differential electron flux measurements from the Rosetta Plasma Consortium (RPC) ${ }^{21}$ (see Extended Data Fig. 1). The neutral and electron observations combined to compute the modelled FUV brightnesses were taken during the same time period as the FUV observations (see Methods). The last three cases (26 December 2015 at 08 UT and 17 April 2016 at 11 UT and 22 UT) attest that in the absence of notable amounts of energetic electrons, as measured in situ by the RPC electron spectrometer (see Extended Data Fig. 1 and Extended Data Table 1), there are nearly no atomic FUV HI or OI emissions detected by the spectrograph (Fig. 2). This demonstrates that there are no other significant sources contributing to the FUV atomic emissions over the shadowed nucleus, beside dissociative excitation of cometary molecules by electrons. In particular, photodissociative excitation of cometary molecules by solar photons do not seem to play any significant role here, as anticipated ${ }^{4}$.

The two 2014 cases (29 Nov at 18:00 UT, 10 Dec at 22:02 UT) correspond to a nadir pointing when Rosetta was located above the neck of the bi-lobed nucleus (Table 1). Comparing observed and modelled OI FUV brightnesses for these two cases, for which a pure water coma is assumed in the absence of in situ gas composition measurements, shows that the observed OI FUV brightnesses are consistent with dissociative excitation of a nearly-pure water coma (Fig. 2-b). This confirms earlier findings that the coma over the neck is primarily composed of water ${ }^{4,18,19}$. In 
this concave region, the outgassing is very active ${ }^{19}$ and emanates in many directions, enhanced by self-illumination during low subsolar latitudes ${ }^{22}$. It is also difficult to derive the detailed activity of the surface in the neck. As a result, the water column density used as input to the model cannot be straightforwardly derived from the number density measured at Rosetta (combined with a simple extrapolation). It is instead set to give the modelled HI Ly $\beta$ brightness in agreement (within 4\%) with the observed one (Fig. 2a and Table 1). The column density of $(3.8 \pm 0.8) \times 10^{15} \mathrm{~cm}^{-2}$, obtained for the 29 November 2014 case, is consistent with the value of $(4.6 \pm 0.3) \times 10^{15} \mathrm{~cm}^{-2}$ derived from Visual InfraRed Thermal Spectrometer (VIRTIS) ${ }^{23}$ observations (see Methods for details). The sensitivity of the OI modelled brightnesses by adding small amounts of $\mathrm{O}_{2}$, $\mathrm{CO}$, or $\mathrm{CO}_{2}$ to the assumed pure water coma is discussed in the Methods section.

In order to establish the origin of the energetic electrons responsible for the FUV auroral emissions, the multi-instrument analysis is applied to limb viewing (see Methods). In that configuration, the FUV spectrograph is staring off nadir at the cometary coma and observing FUV emissions produced in a region of the coma not located between the cometary nucleus and Rosetta. By linking FUV emissions from such a remote region with the emission frequency derived from in-situ electron flux measurements at Rosetta, we are assessing whether energetic electrons are accelerated/heated locally, or they have a large-scale external origin (e.g., hemispheric scale or more). In the former case, the FUV emissions should not be correlated with the energetic electrons, while in the latter, they should be. Without direct measurements of the detailed neutral composition in the remote region observed, the analysis is only applied to $\mathrm{HI} \mathrm{Ly} \beta$ which is solely driven by water. The modelled brightness is derived by multiplying the water column density deduced from 
Microwave Instrument on the Rosetta Orbiter (MIRO) ${ }^{24}$ measurements and VIRTIS infrared observations (coincident with the FUV observation periods), with the HI Ly $\beta$ emission frequency derived from simultaneous in situ RPC electron flux measurements at Rosetta. Two limb-viewing intervals of two days in October 2014 have been analysed (Tables 1 and 2).

Past studies looked at the correlation between the limb brightness in HI Ly $\beta$ from Alice FUV spectrograph and the water column density from VIRTIS infrared spectrometer ${ }^{7}$ and at the correlation between the limb brightness in OI $1356 \AA$ from Alice and the energetic electron density from $\mathrm{RPC}^{25}$. In contrast, here the observed FUV brightness is quantitatively compared with the modelled brightness driven by simultaneous in situ observations of the energetic electron flux from RPC (taking into account the energy distribution of the electrons) and by the water column density measured remotely from Rosetta.

Comparing the HI Ly $\beta$ calculated (blue) and observed (magenta) brightnesses on 18-19 October 2014 (Fig. 3-a) and 22-23 October 2014 (Fig. 3-b) confirms that overall the prime source of the HI Ly $\beta$ emissions is the dissociative excitation of water. There is a good agreement in terms of both magnitude and variability. The relative difference in magnitude is $30 \% \pm 21 \%$ over all periods ( $13 \% \pm 6 \%$ for $\mathrm{P} 3)$ on $18-19$ October 2014 ; it is $22 \% \pm 18 \%$ over all periods $(11 \% \pm 10 \%$ for P3) on 22-23 October 2014. The contribution from resonance scattering driven by the interplanetary medium along the line of sight has been subtracted and amounts to $\sim 1.5$ Rayleigh, while the contribution from the coma is negligible (see Methods). For a given time, the brightness averaged over the rows at the centre of the slit is shown with a dot, while the vertical, light pink bar extends from 
the brightness from rows looking closest to the nucleus (upper bound) to the brightness from rows farthest away from the nucleus (lowest bound) for selected row ranges (see Table 1). The width of the pink bars corresponds to the FUV observation integration time (10 min). The observed limb brightnesses have a $\pm 30 \%$ uncertainty, shown with vertical, thin, magenta lines for three times on each panel.

The very good agreement between the observed and modelled brightnesses in Fig. 3 attests that the energetic electron fluxes measured at Rosetta are consistent with those driving the FUV emissions: the energetic electrons are not locally accelerated/heated. As the water column density is fixed over each FUV observation period $\mathrm{P} x$ (Table 2), the variations in the modelled brightness during $\mathrm{P} x$ is only driven by the variation in the RPC electron fluxes. The very good correlation between the observed and modelled brightness variations includes the overall decrease during P2 on 18 October 2014, the sharp intensification at 16:30 UT and the drop at 21 UT on 22 October 2014, and the decline over P4 on 23 October 2014. The sharp intensification at 16:30 UT, seen in both the modelled and the observed brightnesses, coincides with a large increase in the local plasma density and is associated with the arrival of a solar event ${ }^{26}$. The mean energy and number density of the energetic electrons increase suddenly, which yields an enhancement in both the emission and ionisation frequencies ${ }^{27}$.

Finally, though photoelectrons are present along the line of sight, they cannot constitute the bulk of the energetic electrons responsible for the FUV emissions. The source of the energetic population must be external, as attested by the variability observed in the RPC electron differential flux 
over the limb-viewing periods. Additional evidence is the anti-correlation between the electronimpact ionisation frequency and the local outgassing rate observed away from perihelion ${ }^{27,28}$.

The Rosetta multi-instrument analysis linking coincident particle, neutral gas, and FUV emission datasets together shows that the FUV emissions over the shadowed nucleus observed at large heliocentric distances are dominantly produced by the dissociative excitation of cometary molecules by energetic electrons. The auroral FUV OI emissions at Ganymede ${ }^{9,10}$ and at Europa ${ }^{11}$ are produced by the same type of excitation, while at $\operatorname{Earth}^{29}$ and Venus ${ }^{30}$ they are primarily induced by electron impact on atomic oxygen. However, the source of the energetic electrons is very different at comet $67 \mathrm{P}$ - subject to the interplanetary magnetic field frozen into the solar wind - compared with the ones at the Galilean moons, which are embedded in the intense magnetic field of Jupiter. The energetic electrons, found to be inducing the FUV emissions at comet 67P at large heliocentric distances, were already found to produce most of the ionisation in the coma ${ }^{27}$. They are hence responsible for the presence of a cometary plasma, denser (though colder) than the ambient solar wind, around the nucleus.

Applied to the limb viewing, the multi-instrument analysis demonstrates that the main source of the energetic electrons is not local (hence not photoelectrons as originally thought ${ }^{4,7}$ ). Based on the definition proposed for auroral emissions, this reveals the auroral nature of the FUV atomic emissions. We show that the source of energetic electrons involves a large-scale acceleration mechanism. This finding is consistent with a particle-in-cell simulation applied to a weakly-outgassing comet $^{31}$ (Fig. 4). The self-consistent simulation shows that solar-wind electrons (red dots) undergo 
acceleration primarily along the draped magnetic field lines when they fall into a potential well as they get closer to the cometary nucleus (trajectories color-coded by the electron energy in Fig. 4). This potential well is produced by an ambipolar electric field generated by the cometary plasma and resulting from the large electron pressure gradient ${ }^{31,32}$. This result confirms the original finding ${ }^{33}$ that the observed energetic electron fluxes are too intense and energetic to be explained by unperturbed photoelectrons or unperturbed solar-wind electrons, though they are consistent with the presence of an ambipolar electric field. At Earth, ambipolar electric fields (set up by electron pressure gradients between the cold, dense, ionospheric plasma and the hot, tenuous, magnetospheric plasma) are at least sometimes significant contributors to the large-scale, quasi-stationary, fieldaligned electric fields observed in the auroral (upward field-aligned current) regions ${ }^{34}$. Similar to what is observed at comet 67P, these large-scale electric fields observed at Earth are responsible for the electron acceleration along the draped magnetic field lines. More generally, just like for discrete aurorae at Earth and Mars ${ }^{15,35}$ (which result from the interaction of the terrestrial magnetosphere and the martian remanent crustal magnetic field with the solar wind), we show that the energetic electrons at comet $67 \mathrm{P}$ are accelerated by large-scale electric fields arising from the interaction of the cometary plasma with the solar wind. Lacking an intrinsic magnetic field, the cometary aurora is diffuse, while the terrestrial and martian discrete aurorae are spatially confined. In contrast to the martian diffuse aurora ${ }^{36}$, it occurs even in the absence of solar energetic particle outbursts. While aurora is a universal process, the combination of the excitation process (the same as at Ganymede and Europa) and of the particle acceleration process (resulting from the interaction of the solar wind with the body through electric field acceleration, as for the discrete aurorae 
at Earth and Mars) renders the FUV auroral emissions at comet 67P unique. The discovery of the presence of cometary auroral emissions induced by solar-wind electrons at large heliocentric distances offers the opportunity to use FUV emissions as a probe of the space environment at a comet location: observations of OI $1356 \AA$ (emission not affected by resonance fluorescence) could be used as a proxy for solar-wind electron variability, which would be highly relevant for space weather applications.

\section{References}

1. Taylor, M. G. G. T., Altobelli, N., Buratti, B. J. \& Choukroun, M. The Rosetta mission orbiter science overview: the comet phase. Philos. Trans. R. Soc. A 375, 20160262 (2017).

2. Glassmeier, K.-H., Boehnhardt, H., Koschny, D., Kührt, E. \& Richter, I. The Rosetta Mission: Flying Towards the Origin of the Solar System. Space Sci. Rev. 128, 1-21 (2007).

3. Stern, S. A. et al. Alice: The Rosetta Ultraviolet Imaging Spectrograph. Space Sci. Rev. 128, 507-527 (2007).

4. Feldman, P. D. et al. Measurements of the near-nucleus coma of comet 67P/ChuryumovGerasimenko with the Alice far-ultraviolet spectrograph on Rosetta. Astron. Astrophys. 583, A8 (2015).

5. Feldman, P. D. et al. The Nature and Frequency of the Gas Outbursts in Comet 67P/Churyumov-Gerasimenko Observed by the Alice Far-ultraviolet Spectrograph on Rosetta. Astrophys. J. Lett. 825, L8 (2016). 
6. Feldman, P. D. et al. FUV Spectral Signatures of Molecules and the Evolution of the Gaseous Coma of Comet 67P/Churyumov-Gerasimenko. Astron. J. 155, 9 (2018).

7. Chaufray, J.-Y. et al. Rosetta Alice/VIRTIS observations of the water vapour UV electroglow emissions around comet 67P/Churyumov-Gerasimenko. Mon. Not. R. Astron. Soc. 469, S416S426 (2017).

8. Bieler, A. et al. Abundant molecular oxygen in the coma of comet 67P/ChuryumovGerasimenko. Nature 526, 678-681 (2015).

9. Feldman, P. D. et al. HST/STIS Ultraviolet Imaging of Polar Aurora on Ganymede. Astrophys. J. 535, 1085-1090 (2000).

10. Molyneux, P. M. et al. Hubble Space Telescope Observations of Variations in Ganymede's Oxygen Atmosphere and Aurora. J. Geophys. Res. Space Phys. 123, 3777-3793 (2018).

11. Roth, L. et al. Transient Water Vapor at Europa's South Pole. Science 343, 171-174 (2014).

12. Lupu, R. E., Feldman, P. D., Weaver, H. A. \& Tozzi, G.-P. The Fourth Positive System of Carbon Monoxide in the Hubble Space Telescope Spectra of Comets. Astrophys. J. 670, 1473-1484 (2007).

13. Galand, M. \& Chakrabarti, S. Auroral Processes in the Solar System. Washington DC American Geophysical Union Geophysical Monograph Series 130, 55 (2002).

14. Drossart, P. et al. Detection of $\mathrm{H}_{3}{ }^{+}$on Jupiter. Nature 340, 539-541 (1989).

15. Bertaux, J.-L. et al. Discovery of an aurora on Mars. Nature 435, 790-794 (2005). 
16. Hallinan, G. et al. Magnetospherically driven optical and radio aurorae at the end of the stellar main sequence. Nature 523, 568-571 (2015).

17. Lisse, C. M. et al. Discovery of X-ray and Extreme Ultraviolet Emission from Comet C/Hyakutake 1996 B2. Science 274, 205-209 (1996).

18. Hässig, M. et al. Time variability and heterogeneity in the coma of 67P/ChuryumovGerasimenko. Science 347, aaa0276 (2015).

19. Migliorini, A. et al. Water and carbon dioxide distribution in the 67P/ChuryumovGerasimenko coma from VIRTIS-M infrared observations. Astron. Astrophys. 589, A45 (2016).

20. Balsiger, H. et al. Rosina Rosetta Orbiter Spectrometer for Ion and Neutral Analysis. Space Sci. Rev. 128, 745-801 (2007).

21. Carr, C. et al. RPC: The Rosetta Plasma Consortium. Space Sci. Rev. 128, 629-647 (2007).

22. Keller, H. U. et al. Insolation, erosion, and morphology of comet 67P/ChuryumovGerasimenko. Astron. Astrophys. 583, A34 (2015).

23. Coradini, A. et al. VIRTIS: An Imaging Spectrometer for the Rosetta Mission. Space Sci. Rev. 128, 529-559 (2007).

24. Gulkis, S. et al. MIRO: Microwave Instrument for Rosetta Orbiter. Space Sci. Rev. 128, 561-597 (2007). 
25. Noonan, J. W. et al. Ultraviolet Observations of Coronal Mass Ejection Impact on Comet 67P/Churyumov-Gerasimenko by Rosetta Alice. Astron. J. 156, 16 (2018).

26. Witasse, O. et al. Interplanetary coronal mass ejection observed at STEREO-A, Mars, comet 67P/Churyumov-Gerasimenko, Saturn, and New Horizons en route to Pluto: Comparison of its Forbush decreases at 1.4, 3.1, and 9.9 AU. J. Geophys. Res. Space Phys. 122, 7865-7890 (2017).

27. Heritier, K. L. et al. Plasma source and loss at comet 67P during the Rosetta mission. Astron. Astrophys. 618, A77 (2018).

28. Galand, M. et al. Ionospheric plasma of comet $67 \mathrm{P}$ probed by Rosetta at 3 au from the Sun. Mon. Not. R. Astron. Soc. 462, S331-S351 (2016).

29. Germany, G. A., Torr, M. R., Torr, D. G. \& Richards, P. G. Use of FUV auroral emissions as diagnostic indicators. J. Geophys. Res. Space Phys. 99, 383-388 (1994).

30. Fox, J. L. \& Stewart, A. I. F. The Venus ultraviolet aurora - A soft electron source. J. Geophys. Res. 96, 9821-9828 (1991).

31. Deca, J. et al. Building a weakly outgassing comet from a generalized ohm's law. Phys. Rev. Lett. 123, 055101 (2019).

32. Divin, A. et al. A Fully Kinetic Perspective of Electron Acceleration around a Weakly Outgassing Comet. Astrophys. J. Lett. 889, L33 (2020). 
33. Madanian, H. et al. Suprathermal electrons near the nucleus of comet 67P/ChuryumovGerasimenko at 3 AU: Model comparisons with Rosetta data. J. Geophys. Res. Space Phys. 121, 5815-5836 (2016).

34. Hull, A. J., Bonnell, J. W., Mozer, F. S., Scudder, J. D. \& Chaston, C. C. Large parallel electric fields in the upward current region of the aurora: Evidence for ambipolar effects. J. Geophys. Res. Space Phys. 108, 1265 (2003).

35. Lundin, R. et al. Plasma acceleration above martian magnetic anomalies. Science 311, 980983 (2006).

36. Schneider, N. M. et al. Discovery of diffuse aurora on Mars. Science 350, 0313 (2015).

37. Burch, J. L. et al. RPC-IES: The Ion and Electron Sensor of the Rosetta Plasma Consortium. Space Sci. Rev. 128, 697-712 (2007).

38. Makarov, O. P. et al. Kinetic energy distributions and line profile measurements of dissociation products of water upon electron impact. J. Geophys. Res. Space Phys. 109, A09303 (2004).

39. Mumma, M. J., Stone, E. J., Borst, W. L. \& Zipf, E. C. Dissociative Excitation of Vacuum Ultraviolet Emission Features by Electron Impact on Molecular Gases. III. $\mathrm{CO}_{2}$. J. Chem. Phys. 57, 68-75 (1972).

40. Ajello, J. M. Emission Cross Sections of CO by Electron Impact in the Interval 1260-5000 A. I. J. Chem. Phys. 55, 3158-3168 (1971). 
41. Kanik, I. et al. Electron impact dissociative excitation of $\mathrm{O}_{2}: 2$. Absolute emission cross sections of the $\mathrm{OI}(130.4 \mathrm{~nm})$ and $\mathrm{OI}(135.6 \mathrm{~nm})$ lines. J. Geophys. Res. Planets 108, 5126 (2003).

42. Heritier, K. L. Cometary ionosphere analysis from Rosetta multi-instrument dataset. PhD Thesis Imperial College London (UK) (2018). https : / / doi . org/10 . 25560 /66101.

43. Odelstad, E. et al. Measurements of the electrostatic potential of Rosetta at comet 67P. Mon. Not. R. Astron. Soc. 469, S568-S581 (2017).

44. Eriksson, A. I. et al. RPC-LAP: The Rosetta Langmuir Probe Instrument. Space Sci. Rev. 128, 729-744 (2007).

45. Edberg, N. J. T. et al. Solar wind interaction with comet 67P: Impacts of corotating interaction regions. J. Geophys. Res. Space Phys. 121, 949-965 (2016).

46. Le Roy, L. et al. Inventory of the volatiles on comet 67P/Churyumov-Gerasimenko from Rosetta/ROSINA. Astron. Astrophys. 583, A1 (2015).

47. Gasc, S. et al. Sensitivity and fragmentation calibration of the time-of-flight mass spectrometer RTOF on board ESA's Rosetta mission. Planet. Space Sci. 135, 64-73 (2017).

48. Jorda, L. et al. The global shape, density and rotation of Comet 67P/Churyumov-Gerasimenko from preperihelion Rosetta/OSIRIS observations. Icarus 277, 257-278 (2016). 
49. Bockelée-Morvan, D. et al. First observations of $\mathrm{H}_{2} \mathrm{O}$ and $\mathrm{CO}_{2}$ vapor in comet 67P/Churyumov-Gerasimenko made by VIRTIS onboard Rosetta. Astron. Astrophys. 583, A6 (2015).

50. Dhiri, V. J. F., Vallat, C. \& Kueppers, M. Rosetta: Payload boresight alignment details. ESA 2, 1-11, doi: RO-EST-TN-3305 (2014).

51. Fougere, N. et al. Direct Simulation Monte Carlo modelling of the major species in the coma of comet 67P/Churyumov-Gerasimenko. Mon. Not. R. Astron. Soc. 462, S156-S169 (2016).

52. Biver, N. et al. Long-term monitoring of the outgassing and composition of comet 67P/Churyumov-Gerasimenko with the Rosetta/MIRO instrument. Astron. Astrophys. 630, A19 (2019).

53. Fink, U. et al. Investigation into the disparate origin of $\mathrm{CO}_{2}$ and $\mathrm{H}_{2} \mathrm{O}$ outgassing for Comet 67/P. Icarus 277, 78-97 (2016).

54. Deca, J. et al. Electron and Ion Dynamics of the Solar Wind Interaction with a Weakly Outgassing Comet. Physical Review Letters 118, 205101 (2017).

Acknowledgements Rosetta is a European Space Agency (ESA) mission with contributions from its member states and the National Aeronautics and Space Administration (NASA). We acknowledge the continuous support of the Rosetta teams at the European Space Operations Centre in Darmstadt and at the European Space Astronomy Centre. We acknowledge the staff of CDDP and Imperial College for the use of AMDA and the RPC Quicklook database. This work has benefited from discussions within International Team 402: 
Plasma Environment of Comet 67P after Rosetta at the International Space Science Institute (ISSI) (Bern, Switzerland). We acknowledge PRACE for awarding us access to Curie at GENCI@CEA, France. We warmly thank Nicolas Fougere for his help and advice using the ICES models. Work at Imperial College London was supported by STFC of UK under grant ST/N000692/1 and ST/P002250/1. The Alice team acknowledges support from NASA's Jet Propulsion Laboratory through contract 1336850 to the Southwest Research Institute. MR acknowledges the support of the State of Bern and the Swiss National Science Foundation (200021_165869,200020_182418). J. D. gratefully acknowledges support from NASAs Rosetta Data Analysis Program, Grant No. 80NSSC19K1305, NASA's Solar System Exploration Research Virtual Institute (SSERVI): Institute for Modeling Plasmas, Atmosphere, and Cosmic Dust (IMPACT), and the computational resources provided by the NASA High-End Computing (HEC) Program through the NASA Advanced Supercomputing (NAS) Division at Ames Research Center. We acknowledge PRACE for awarding us access to Curie at GENCI@CEA, France. Work at LPC2E/CNRS was supported by CNES and by ANR under the financial agreement ANR-15-CE31-0009-01. VIRTIS was built by a consortium, which includes Italy, France, and Germany, under the scientific responsibility of the Istituto di Astrofisica e Planetologia Spaziali of INAF, Italy, which also guides the scientific operations. The VIRTIS instrument development, led by the prime contractor Leonardo-Finmeccanica (Florence, Italy), has been funded and managed by ASI, with contributions from Observatoire de Meudon financed by CNES, and from DLR. We thank the Rosetta Science Ground Segment and the Rosetta Mission Operations Centre for their support throughout all the phases of the mission. The VIRTIS calibrated data will be available through the ESAs Planetary Science Archive (PSA) Website (www.rssd.esa.int) and is available upon request until posted to the archive. We thank the following institutions and agencies for support of this work: Italian Space Agency (ASI, Italy) contract number I/024/12/1, Centre National d'Etudes Spatiales (CNES, France), DLR (Germany), NASA (USA) Rosetta Program, and Science and Technology Facilities Council (UK). All ROSINA data are the 
work of the international ROSINA team (scientists, engineers and technicians from Switzerland, France, Germany, Belgium and the US) over the past 25 years, which we herewith gratefully acknowledge.

Author contributions M.G. led the study, performed the multi-instrument analysis, generated Fig. 2 and 3, and wrote the manuscript. P.D.F. identified times of interest for Alice, analysed the FUV dataset, advised on the different emission source mechanisms, and estimated the interplanetary medium contribution. D.B.-M. and Y.-C.C. analysed the VIRTIS-H dataset. N.B. analysed the MIRO dataset. G.R. analysed the VIRTIS-M dataset. M.R. and K.A. (Principal investigator of the ROSINA instrument) provided the ROSINA dataset. They all provided guidance on the interpretation of their respective dataset. J.D. generated Fig. 4 based on the output of a PiC simulation he ran. J.D. and P.H. provided guidance on the PiC simulation interpretation. A.B., P.S. and K.L.H. provided feedback on the multi-instrument analysis. A.B. generated Fig. 1. J.Wm.P. (Principal investigator of the Alice instrument) contributed to the interpretation of the Alice dataset. C.C., A.I.E., and J.B. (all Principal investigators of RPC) provided guidance on the interpretation of the RPC dataset. A.I.E. provided the RPC-LAP dataset. All authors contributed to the interpretation of the results and commented on this manuscript.

Interest declaration The authors declare that they have no competing financial interests.

Correspondence Correspondence and requests for materials should be addressed to M. Galand (email: mgaland@ic.ac.uk).

\section{Methods}

We apply a multi-instrument analysis linking coincident Rosetta electron, neutral gas, and FUV emission observations together (Fig. 1). The measured FUV brightnesses for HI and OI emissions are compared with 
the calculated brightnesses derived from electron and neutral gas measurements. The latter includes in situ measurements from a mass spectrometer as well as remote-sensing sub-mm and infrared observations. The auroral nature that we derive for the FUV emissions is consistent with a particle-in-cell simulation applied to low outgassing comets.

Modelled FUV brightnesses. We calculate the brightness of three atomic emissions, HI Ly $\beta$ line (1026 $\AA$ ) and OI multiplets (1304 $\AA$ and $1356 \AA$ ), for seven cases in nadir viewing over the shadowed nucleus and for two periods of two days in limb viewing (Table 1). The number of cases is restricted by the requirements (1) to have analysed FUV brightness observations, with high enough signal to noise, over the northern hemisphere, (2) for the nadir study, to have the FUV spectrograph viewing along the nadir over the shadowed nucleus and to have simultaneous in situ neutral density and composition measurements (though two cases without neutral composition were included as they were over the nucleus' neck where the coma is known to be almost pure water), (3) for the limb study, to have coincident limb-viewing observations from the FUV spectrograph and from either the sub-mm instrument or one of the infrared sensors. The brightness (in Rayleigh) of an atomic emission $X$ is assumed to be produced by the dissociative excitation of neutral molecules by energetic electrons. It is assessed, as a function of the time $t$, as follows:

$$
B^{X}(t)=10^{-6} \nu^{X}(t) C(t)
$$

where $\nu^{X}$ is the combined frequency (in $\mathrm{s}^{-1}$ ) of dissociative excitation of neutral cometary species which contribute to the production of the atomic emission $X$ and $C$ is the total column density (in $\mathrm{cm}^{-2}$ ), along the line of sight, of these neutral species. As HI Ly $\beta$ is only produced by the dissociation of water, its brightness is derived from the emission frequency of water and the water column density along the line of sight. As the OI emissions are induced by the dissociation of several neutral species, their brightnesses are calculated from the combined emission frequency (defined hereafter) and the total column density of $\mathrm{H}_{2} \mathrm{O}, \mathrm{CO}_{2}, \mathrm{CO}$, 
and $\mathrm{O}_{2}$ along the line of sight. For the nadir viewing, the modelled value provided for each case derives from the average value over all measurements of RPC-Ion and Electron Sensor (IES) ${ }^{37}$ over the observing time of Alice (Fig. 2 and Table 1). For the limb viewing, the modelled values are provided at each time that an energetic electron spectrum of RPC-IES is measured (Fig. 3). The typical time resolution of RPC-IES over the selected limb-viewing days is $4 \mathrm{~min}$.

Electron-impact emission frequency: The emission frequency $\nu_{n}^{X}$ of the atomic emission $X$ (HI Ly $\beta$, OI 1304, OI 1356) associated with the dissociation of the neutral species $n\left(\mathrm{H}_{2} \mathrm{O}, \mathrm{O}_{2}, \mathrm{CO}_{2}, \mathrm{CO}\right)$ is calculated at time $t$ at the location of Rosetta as follows:

$$
\nu_{n}^{X}(t)=\int_{E_{n}^{X}}^{E_{\max }} \sigma_{n}^{X}(E) J_{e}(t, E) \mathrm{d} E
$$

where $\sigma_{n}^{X}(E)$ is the dissociative excitation cross section (in $\mathrm{cm}^{2}$ ) of $n$ by an electron of energy $E$ and $J_{e}(t, E)$ is the differential electron flux (in $\mathrm{cm}^{-2} \mathrm{~s}^{-1} \mathrm{eV}^{-1}$ ) measured at time $t$. We consider cross sections from $\mathrm{H}_{2} \mathrm{O}$ yielding $\mathrm{HI} \mathrm{Ly} \beta$ and $\mathrm{OI}$ emissions ${ }^{38}$, from $\mathrm{CO}_{2}$ yielding OI $1304^{39}$ and OI $1356^{4}$, from CO yielding OI multiplets ${ }^{40}$, and from $\mathrm{O}_{2}$ yielding OI multiplets ${ }^{41}$. $J_{e}$ can be assumed to be constant along the line of sight ${ }^{7,27}$. It is obtained from the electron intensity (in $\mathrm{cm}^{-2} \mathrm{~s}^{-1} \mathrm{eV}^{-1} \mathrm{sr}^{-1}$ ) measured by the RPC-IES, after integrating the latter over elevation and azimuthal angles and assuming isotropy for blind spots due to obstruction or the limited field of view ${ }^{42}$. The differential electron flux is also corrected for the spacecraft potential ${ }^{43}$ - obtained from RPC-LAP ${ }^{44}$ - by applying Liouville's theorem ${ }^{28}$. On 10 December 2014, as no data is available for the spacecraft potential $V_{s c}$, it was set to $-10 \mathrm{~V}$. The arrival of a CIR on 22 October 2014 at 16:30 UT rendered the spacecraft potential very negative but could not be derived from RPC-LAP over the rest of the day and the next day until 06 UT $^{45}$. From 16:30 UT onward on 22 October $2014 V_{s c}$ is set to $-25 \mathrm{~V}$ (part of $\mathrm{P} 1$ and period $\mathrm{P} 2$ ), while on 23 October 2014 which was less disturbed, it is set to $-15 \mathrm{~V}$ (periods P3 and P4). The RPC-IES dataset is not reliable after 17:25 UT on 22 October 2014 
for about 15-20 min, so it is disregarded. The energy $E_{\max }$ is the maximum energy considered which is set to $200 \mathrm{eV}$; beyond this value, the signal is primarily at the background level. We check that the emission frequency is not sensitive to the choice of a higher value for $E_{\max }$, testing it up to $400 \mathrm{eV}$. The energy $E_{n}^{X}$ represents the energy threshold of the dissociative excitation process; its value is $17 \mathrm{eV}$ for $\mathrm{HI} \mathrm{Ly} \beta$ from the dissociation of $\mathrm{H}_{2} \mathrm{O}$; it varies between $14-15 \mathrm{eV}\left(\mathrm{H}_{2}, \mathrm{O}_{2}\right)$ to $20-21 \mathrm{eV}\left(\mathrm{CO}, \mathrm{CO}_{2}\right)$ for the OI emissions. When $V_{s c}$ is very negative, the corrected differential electron flux from RPC-IES starts at an energy $E_{\min }$ above the ionisation threshold. In that case, it is extrapolated towards lower energies assuming a constant value equal to the measured value at $E_{\min }$. Figure ?? shows two examples of differential electron fluxes, as a function of energy, measured by the RPC-IES electron spectrometer and used in the nadir study: one taken at 11:47 UT (orange crosses) during the FUV observation period on 29 March 2015 starting at 11:43 UT and the other taken at 08:35 UT (red pluses) taken during the FUV observation period on 26 December 2015 (Table 1). The differential fluxes are corrected for the spacecraft potential; as, by coincidence, the latter is of the same order in both cases $(-2 \mathrm{~V})$, the spectra start at about the same energy (about $8.5 \mathrm{eV}$ ). By integration, the density of electrons with energies between $10 \mathrm{eV}$ and $200 \mathrm{eV}$ is derived and found to be 30 times higher in the March case than in the December case. The former is associated with a period when significant FUV emissions are detected, while the latter is associated with a period of absence of significant FUV emissions (see Figure 2).

Unlike HI Ly $\beta$ which is only induced by the dissociation of water, OI emissions are produced by the dissociative excitation of all four major species. In that case, it is necessary to assess an effective emission frequency, defined as:

$$
\nu^{X}(t)=\sum_{n} v_{n}(t) \nu_{n}^{X}(t)
$$

where $v_{n}(t)$ is the volume mixing ratio of the neutral species $n$ at time $t$. It is derived from the analysis 
of the ROSINA-DFMS dataset obtained during the observing period of Alice. The data processing and analysis of ROSINA-DFMS to derive the neutral composition are described in Le Roy et al. ${ }^{46}$. The neutral composition is assumed to be constant in the nadir-viewing column of the coma. When it is not available (e.g., 2014 nadir-viewing cases), the forward modelling is performed for a pure-water coma. The closest DFMS measurements to one of the 2014 nadir-viewing cases was made on 10 December 2014 at 22 UT. It shows that, after water, $\mathrm{O}_{2}$ was the second most abundant species (3\%), followed by $\mathrm{CO}(2 \%)$ and $\mathrm{CO}_{2}$ $(0.7 \%)$ with a decreasing trend (with respect to water) observed from 20 UT to 22 UT. This trend suggests that the mixing ratios of the minor species during the Alice observation window (22:02-23:13 UT) are likely to be smaller than those listed above. The modelled OI brightnesses for pure water are shown in Fig. $2 \mathrm{~b}$. For the 10 December 2014 case, while the OI 1304 brightnesses agree within the uncertainty, the OI 1356 brightness is $\sim 45 \%$ lower compared with the Alice brightness (which has an absolute calibration uncertainty of $\pm 20 \%$ ). Adding $0.5 \%$ of $\mathrm{O}_{2}$ (relative to water) brings the modelled OI brightness within $5 \%$ of the Alice OI 1356 brightness (electron impact on $\mathrm{O}_{2}$ being efficient to produce OI $1356^{41}$ ), without affecting significantly the OI 1304 modelled brightness (which remains within $\sim 15 \%$ of the observed brightness), as OI 1304 is dominantly produced through the dissociation of water ${ }^{38}$. Adding $2 \%$ of $\mathrm{CO}\left(\right.$ or $1 \%$ of $\mathrm{CO}_{2}$ ) to the $\mathrm{H}_{2} \mathrm{O}-\mathrm{O}_{2}$ coma, the OI 1356 modelled brightness is higher compared with the Alice brightness by 3-9\% (12-16\%), respectively, but remains within the uncertainties of the observed value.

Nadir column density: For nadir viewing, the total neutral column density along the line of sight corresponds to the number of molecules per unit area in the column between the Rosetta spacecraft and the surface of the nucleus. By default, the column density is derived from the total neutral density $n_{\text {tot }}^{\text {COPS }}(t, r)$ measured at time $t$ at the Rosetta cometocentric distance $r_{R}$, by the ROSINA-Comet Pressure Sensor (COPS ${ }^{20}$, after correction ${ }^{47}$ for neutral composition inferred from ROSINA-DFMS. We assume a $r^{-2}$-dependence in 
cometocentric distance $r$ for the number density down to the surface, as justified by observations ${ }^{8,18}$. This means that for nadir viewing, the column density at time $t$ is:

$$
C^{\mathrm{COPS}}(t)=n_{t o t}^{\mathrm{COPS}}\left(t, r_{R}\right) \frac{\left(r_{R}-r_{S}\right) r_{R}}{r_{S}}
$$

where $r_{S}$ is the cometocentric distance of the nucleus' surface, assumed here to be a mean value of $1.7 \mathrm{~km}^{48}$. Values derived for the column density are given in Table 1 for the four 2015-2016 nadir cases and in Table 3 for the two times selected in Fig. ??.

For the two 2014 nadir cases, which correspond to cases above the highly active neck of the bi-lobed nucleus $^{48}$, the geometry of the surface means that the gas is emitted in many directions with enhanced level due to self-illumination ${ }^{22}$. It is not realistic to infer the column density close to the nucleus from measurements of the neutral density at Rosetta. Instead, the water column density is derived from the comparison between the observed and modelled HI Ly $\beta$ brightnesses (Table 1).

Nadir column density on 29 November 2014: Based on the HI Ly $\beta$ analysis, we derive a value of (3.8 \pm $0.8) \times 10^{15} \mathrm{~cm}^{-2}$ (uncertainty linked to the $20 \%$ uncertainty in the observed nadir HI Ly $\beta$ brightness) for the water column density for the 29 November 2014 case and used it to drive the model. This value is consistent with the water column density value of $(4.6 \pm 0.3) \times 10^{15} \mathrm{~cm}^{-2}$ obtained from the high spectral-resolution single-aperture spectrograph, VIRTIS- $\mathrm{H}^{49}$ ( $\mathrm{H}$ for High resolution) during the Alice observation period on the same day. It should be noted that there may be a slight difference in the close-up regions seen by Alice and VIRTIS-H at such a small distance from the nucleus, as highlighted by comparing their boresights and fields of view ${ }^{50}$ : Alice brightness is from bins $15-17$ along the slit (Table 1), while VIRTIS-H aperture is closest to the bin $14 / 15$ junction; the field of view of VIRTIS-H $\left(0.03^{\circ} \times 0.1^{\circ}\right)^{49}$ is slightly smaller than that associated with a bin of Alice $\left(0.05^{\circ} \times 0.3^{\circ}\right)^{6}$. There is also a slight difference in the time period of the two observation sets: 17:57-18:22 UT (VIRTIS-H), 18:00-18:40 UT (Alice). The derived value for 
the water column density is also close to the value of $6 \times 10^{15} \mathrm{~cm}^{-2}$ deduced from the DSMC model for the region of interest ${ }^{51}$. As expected over the neck region, the water column density extrapolated from the neutral density measurements at Rosetta from ROSINA and assuming a mean cometocentric distance of the nucleus' surface of $1.7 \mathrm{~km}^{48}$ is significantly smaller than the one deduced from VIRTIS-H (by $84 \%$ ) and the one derived from $\mathrm{HI} \operatorname{Ly} \beta(82 \%)$.

Limb column density: For limb viewing, the column to consider along the viewing direction stretches from the Rosetta spacecraft to infinity. In practice, it extends up to where the coma is dense enough to emit significant emissions to be detected by the remote-sensing instruments. Only HI Ly $\beta$, induced by the dissociation of water, is analysed for limb cases. The water column density is derived from the Rosetta sub-mm MIRO instrument and from the IR VIRTIS instrument suite. Microwave emissions at wavelengths near $0.53 \mathrm{~mm}$ emitted by $\mathrm{H}_{2}^{18} \mathrm{O}$ and observed by the high-resolution spectrograph from $\mathrm{MIRO}^{24}$ were analysed in order to derive the water column density ${ }^{52}$. An expansion velocity of $0.68 \mathrm{~km} \mathrm{~s}^{-1}$ was assumed for the analysis of the limb observations. The $\nu_{3}$ vibrational band of water near $2.7 \mu \mathrm{m}$, the strongest vibrational band observed in cometary infrared spectra, was detected by VIRTIS ${ }^{23}$. Emission intensities from the high spectral-resolution single-aperture spectrograph, VIRTIS-H, were analysed in the $2.61-2.73 \mu \mathrm{m}$ range in order to derive water column density. The data processing and analysis of such a dataset are described in Bockelée-Morvan et al. ${ }^{49}$. Emission intensities from the infrared channel of the medium-resolution imaging spectrometer, VIRTIS-M (M for Mapper), were analysed by integrating over the 2.6-2.8 $\mu \mathrm{m}$ band after subtracting the background continuum ${ }^{19,53}$.

The water column density values used for calculating the FUV HI Ly $\beta$ brightnesses during each limbviewing period are listed in the fourth column in Table 2 along with the values observed by the MIRO instrument in the sub-mm (fifth column), by the VIRTIS IR high-resolution spectrograph (sixth column) and 
medium-resolution imaging spectrometer (seventh column). For period P3 of Alice observations (around midnight on 18 October 2014), measurements from all three remote sensors are available and agree very well. For the other periods, when available the water column densities derived from the IR mediumresolution imaging spectrometer are consistent with those derived from the sub-mm observations. As the water column density derived from the sub-mm instrument has the lowest uncertainty, we set the value used for the limb-viewing calculation to its mean value.

Observed FUV brightnesses. The FUV brightnesses are derived from the Alice imaging spectrograph ${ }^{3}$ for nadir and limb-staring viewings. Among $\mathrm{HI}$ lines, $\mathrm{Ly} \beta$ is preferable to the stronger Ly $\alpha$ for the present study due to the complexity of instrumental effects for Alice measurements. For limb viewing, the signal is also affected by the resonance scattering of the interplanetary H Lyman series, which is at least 300 times brighter in HI Ly $\alpha$ than in $\mathrm{HI} \operatorname{Ly} \beta$. Even for nadir viewing over the shadowed nucleus, where such a contribution is not significant, the Ly $\alpha$ sensitivity varies by a factor of 2 along the slit due to the uneven photocathode deposited on the microchannel plate detector in the region of $\mathrm{Ly} \alpha^{3}$.

For each bin along the slit, an individual spectrum is obtained after a time integration of typically $10 \mathrm{~min}$. The slit has a dog-bone shape with a narrow, central region of width $0.05^{\circ}$ and of length $2^{\circ 3}$, spanning from bins 12 to $18\left(0.3^{\circ} / \mathrm{bin}\right)$. The brightnesses for nadir viewing and the main brightnesses for limb viewing (magenta dots in Figure 3) are obtained from the central part of the narrow region of the slit, which provides the best spectral resolution possible with Alice. The central bin of the narrow region of the slit, bin 15 , represents the closest bin to nadir when the $z$ axis is nadir. All nadir viewing brightnesses are associated with a bin range including bin 15 (see Table 1). The only exception is 26 December 2015 which is slightly off nadir and, to a lesser extent, 17 April 2016. For limb viewing, beside the brightness around the slit's centre, two other brightnesses are given at each time, one generated from bins closer to the nucleus and 
another one from bins further away from the nucleus (Table 1).

Once the spectra are co-added over the bin range and the count rate converted into a value in photons $\cdot \mathrm{R}^{-1}$, the spectra are sometimes averaged over time in order to improve the signal-to-noise ratio. This is done for the nadir observations over the shadowed nucleus. This explains why the observing periods, which are the sum of individual exposures, are ranging from 20 min to over 1 h 30 min (Table 1). For the limb viewing, the original 10-min integration has been conserved. After removal of the background derived from spectral regions cleared of strong lines, the brightness is estimated from integration over the atomic emission.

The HI and OI brightnesses for two nadir-viewing cases (29 November 2014 at 18:00 UT and 29 March 2015 at 11:43 UT) have already been published ${ }^{6}$ and further information on the Alice data analysis can be found there. The HI Ly $\beta$ brightnesses for the two limb-viewing cases (18-19 October 2014 and 22-23 October 2014) are updated from Figs. 4 and 5 of Feldman et al. ${ }^{4}$, as since the publication the instrument calibration has been revised. The contribution of resonance scattering from the coma and the interplanetary medium (IPM) is estimated along the line of sight for these two observation periods. The contribution from the coma is assessed to be of the order of $\mathrm{mR}$ assuming a spherically symmetric neutral coma: it can be reliably neglected. The contribution from interplanetary $\mathrm{HI}$ is estimated based on nearly concurrent measurements made at larger off-nadir angles (and during a period of low measured electron flux). The uncertainty on the Alice limb brightnesses, including calibration uncertainty and IPM contribution, is estimated to be $\pm 30 \%$.

Particle-in-cell simulations. To illustrate the large-scale energisation of electrons, we present the results of a 3D fully kinetic particle-in-cell simulation applied to a weakly-outgassing comet at large heliocentric distances $^{54}$. The plasma environment is simulated for an heliocentric distance of $4 \mathrm{AU}$ and an outgassing rate for the cometary nucleus of $10^{25} \mathrm{~s}^{-131}$. The simulation shows that the solar-wind electrons, originally at $\sim 10 \mathrm{eV}$, are accelerated towards the nucleus as they fall into the potential well produced by an ambipolar 
electric field. This electric field is set up by the cometary plasma and is triggered by a strong electron pressure gradient (Fig. 4).

Data Availability: The Rosetta data that support the plots within this paper and other findings of this study are available from the ESA-PSA archive (https://www.cosmos.esa.int/web/psa/rosetta) or the NASA PDS archive (https://pdssbn.astro.umd.edu/data_sb/missions/rosetta/index.shtml)

Code Availability: iPIC3D is publicly available on GitHub (https://github.com/iPIC3D/iPIC3D; Apache License 2.0). 
Figure 1: Multi-instrument approach applied to analyse FUV atomic emissions. Overview of the generation of auroral emissions through the dissociative excitation of cometary molecules by energetic (10-200 eV) electrons. A multi-instrument approach is applied to confirm the origin of the FUV emissions by linking (a) the energetic electrons measured in situ by the Rosetta Plasma Consortium (RPC) ${ }^{21}$ electron spectrometer ${ }^{37}$, (b) the cometary molecules observed in situ by the Rosetta Orbiter Spectrometer for Ion and Neutral Analysis (ROSINA) ${ }^{20}$ and remotely by the Microwave Instrument on the Rosetta Orbiter (MIRO) ${ }^{24}$, and the Visual InfraRed Thermal Spectrometer (VIRTIS) ${ }^{23}$, and (c) the FUV atomic emissions detected by the Alice FUV spectrograph ${ }^{3}$.

Figure 2: Nadir-viewing analysed cases. Nadir-viewing FUV brightnesses observed (magenta) and calculated (black) from a combination of coincident neutral gas and electron measurements (a) for HI Ly $\beta$ line and (b) for OI $1304 \AA$ (filled circles) and OI $1356 \AA$ (filled triangles) multiplets. The magenta vertical bars include $20 \%$ uncertainty in the observed brightness values and $\pm 1 \sigma$ standard deviation resulting from the spread over the spatial rows in the extracted spectrum. The black vertical bars represent the variability in Rosetta in situ electron fluxes over the FUV observing time combined, for the OI brightnesses, with $20 \%$ in Rosetta in situ neutral composition uncertainty (except for the 2014 cases for which a pure water coma is assumed over the neck in the absence of coincident neutral composition observations). Measured and modelled points are offset for a given time for visibility. 
Figure 3: Limb-viewing analysed cases. Time series of limb-viewing observed (magenta) and calculated (blue) HI Ly $\beta$ brightnesses (a) on 18-19 October 2014 and (b) on 22-23 October 2014. The model is driven by Rosetta in situ electron measurements and by the water column density derived from Rosetta remote-sensing sub-mm and IR observations (see Table 2). The observed FUV brightness is averaged over the rows at the centre of the slit (dot) and its uncertainty is $\pm 30 \%$ (vertical, thin, magenta lines for three times on each panel). The vertical, light pink bar shows the variation along the slit; its width corresponds to the FUV spectrograph integration time (10 min).

Figure 4: Source of the energetic electrons. Trajectories of solar-wind electrons inducing the FUV aurora around comet 67P. They undergo acceleration through the ambipolar electric field set up by the cometary plasma. The electron trajectories are shown with lines colour-coded by energy and the ambipolar electric field acting on electrons $\left(-\mathbf{E}_{a m b i}\right)$, with green arrows. They are output from a 3D fully kinetic particle-in-cell simulation applied to a weakly-outgassing comet $^{31}$. The upstream solar wind flows along $+X$ (towards the right), the upstream interplanetary magnetic field points along $+\mathrm{Y}$ (upward), and $\mathrm{Z}$ is complementing the orthogonal coordinate system (out of the plane). The nucleus is not to scale.

Figure 5: Extended Data Fig.1. Examples of differential electron fluxes measured by the RPC-IES electron spectrometer. The fluxes were observed at 11:47 UT on 29 March 2015 (orange crosses) and at 08:35 UT on 26 December 2015 (red pluses) during two nadir-viewing FUV observation periods. The fluxes are corrected for the spacecraft potential ${ }^{28}(-2 \mathrm{~V})$. By integration, the number density and mean energy of electrons with energies between $10 \mathrm{eV}$ and $200 \mathrm{eV}$ are derived and given in Extended Data Table 1. 
Table 1: Details on the analysed cases. For nadir viewing, are given: selected day, Alice FUV spectrograph observation start time $t_{0}$ and duration $\Delta t$ (sum of all integration times used), bin number range used along the FUV spectrograph slit, heliocentric distance $r_{h}$, Rosetta cometocentric distance $r_{R}$ and sub-spacecraft latitude at $\mathrm{t}_{0}$, and column density $C$ between Rosetta and the nucleus' surface. For limb viewing, are given: selected day, range of bins along the FUV spectrograph slit from closest to the nucleus, centre of the slit, to furthest from the nucleus, distances $r_{h}$ and $r_{R}$, FUV spectrograph off-nadir viewing angle, and integration time $\Delta t$.

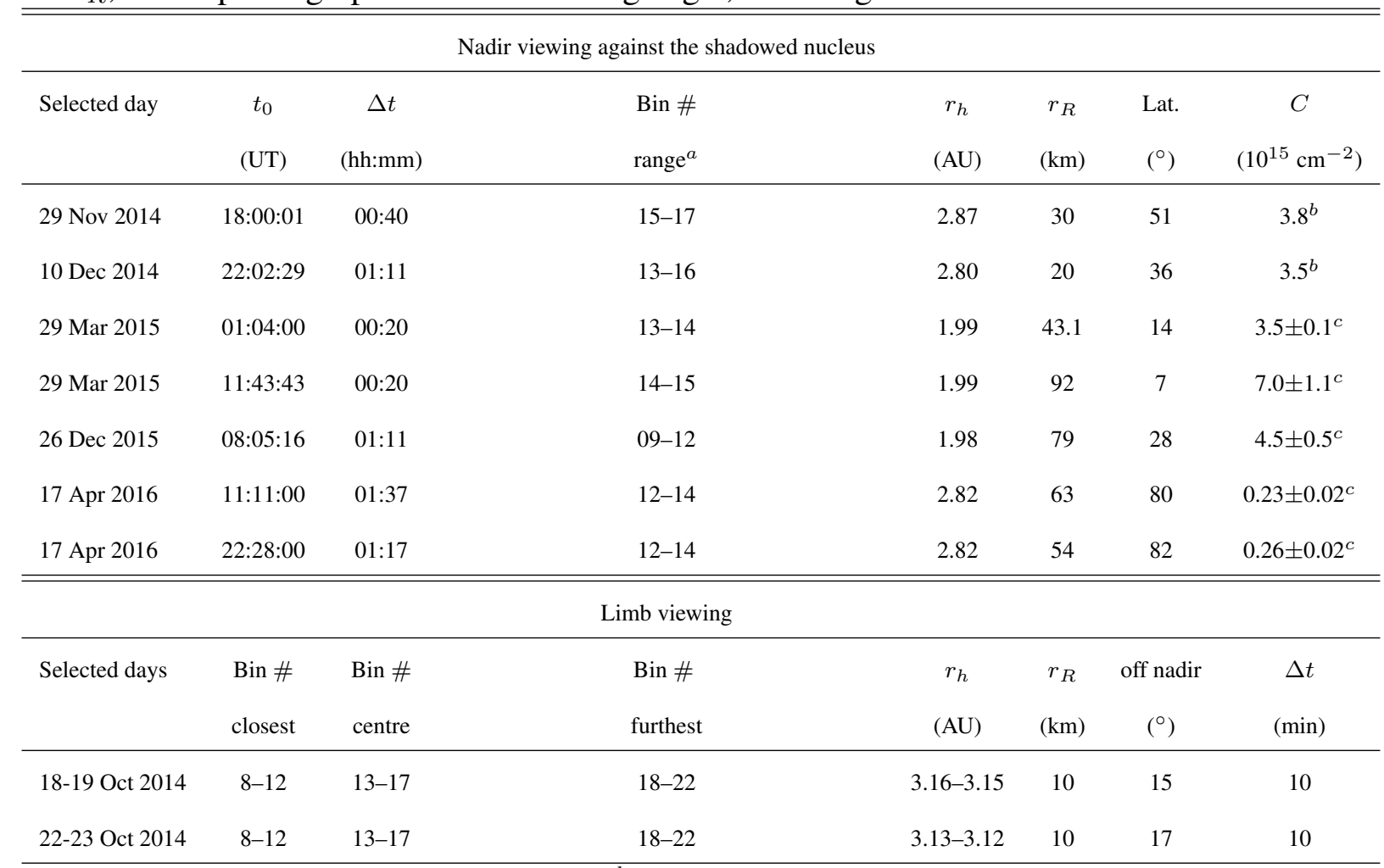

${ }^{a}$ The centre of the slit, closest to nadir, is bin $15 .{ }^{b}$ The total column density is deduced from HI Ly $\beta$ observations assuming a water pure coma (see text). ${ }^{c}$ The total column density is derived from the total number density $n_{\text {tot }}^{\text {COPS }}$ measured by the ROSINA-COPS pressure gauge, assuming a mean cometocentric distance for the nucleus' surface of $1.7 \mathrm{~km}^{48}$ and the neutral composition derived from the ROSINA-DFMS mass spectrometer. 
Table 2: Water column density for the limb cases. Are given the period $\mathrm{P} x$ selected, the date, the time range of $\mathrm{P} x$ (corresponding to the sub-mm observing period), the value $C^{\text {limb }}$ of the water column density used for the calculation of the FUV brightness (see Figure 3), based on the measurements of the column density by the MIRO instrument in the sub-mm $\left(C^{\mathrm{MIRO}}\right)$, by the IR high-resolution spectrograph $\left(C^{\text {VIRTIS-H }}\right)$ and by the medium-resolution imaging spectrometer $\left(C^{\text {VIRTIS-M }}\right)$. When no data is available, the column density entry is left blank. The remote-sensing IR measurements are made over approximately the same time range as the sub-mm observations (third column), though there are sometimes some departures in terms of the start or end times (up to $15 \mathrm{~min}$ ) between instruments.

\begin{tabular}{|c|c|c|c|c|c|c|}
\hline \multicolumn{7}{|c|}{ 18-19 December 2014} \\
\hline \multirow[t]{2}{*}{ Selected period } & \multirow[t]{2}{*}{ Day } & \multirow{2}{*}{$\begin{array}{c}\text { Time range } \\
\text { (UT) }\end{array}$} & \multirow{2}{*}{$\begin{array}{c}C^{\operatorname{limb}} \\
\left(10^{15} \mathrm{~cm}^{-2}\right)\end{array}$} & \multirow{2}{*}{$\begin{array}{c}C^{\mathrm{MIRO}} \\
\left(10^{15} \mathrm{~cm}^{-2}\right)\end{array}$} & \multirow{2}{*}{$\begin{array}{c}C^{\text {VIRTIS-H }} \\
\left(10^{15} \mathrm{~cm}^{-2}\right)\end{array}$} & \multirow{2}{*}{$\begin{array}{c}C^{\text {VIRTIS-M }} \\
\left(10^{15} \mathrm{~cm}^{-2}\right)\end{array}$} \\
\hline & & & & & & \\
\hline $\mathrm{P} 1$ & 18 Dec 2014 & $15: 30-17: 40$ & 1.4 & $1.41 \pm 0.07$ & & $1.6 \pm 0.7$ \\
\hline $\mathrm{P} 2$ & 18 Dec 2014 & $18: 45-21: 40$ & 2.0 & $2.04 \pm 0.07$ & & $2.1 \pm 0.9$ \\
\hline P3 & 18-19 Dec 2014 & $23: 40-01: 40$ & 2.9 & $2.87 \pm 0.09$ & $2.8 \pm 0.2$ & $3.4 \pm 1.4$ \\
\hline P4 & 19 Dec 2014 & $02: 50-05: 40$ & 1.1 & $1.14 \pm 0.06$ & & \\
\hline \multicolumn{7}{|c|}{ 22-23 December 2014} \\
\hline $\mathrm{P} 1$ & 22 Dec 2014 & $15: 10-17: 40^{a}$ & 1.9 & $1.85 \pm 0.08$ & & $2.0 \pm 0.8$ \\
\hline $\mathrm{P} 2$ & 22 Dec 2014 & $18: 45-21: 40^{b}$ & 1.7 & $1.68 \pm 0.07$ & & $1.9 \pm 0.8$ \\
\hline P3 & 22-23 Dec 2014 & $23: 40^{b}-01: 40$ & 1.4 & $1.38 \pm 0.10$ & & $2.1 \pm 0.9$ \\
\hline P4 & 23 Dec 2014 & $02: 40-05: 40$ & 1.1 & $1.10 \pm 0.06$ & & $1.2 \pm 0.5$ \\
\hline
\end{tabular}

${ }^{a}$ The HI Ly $\beta$ brightnesses over P1 on 22 December 2014 are calculated up to 17:25 UT (see Figure 3b), as the differential flux from the electron spectrometer is not reliable for the rest of P1. ${ }^{b}$ The HI Ly $\beta$ brightnesses over P2 and P3 on 22 December 2014 are calculated up to 22:00 UT and from 23:10 UT, respectively (see Figure 3b) in order to show the trend driven by the variability in the measured electron differential flux. 
Table 3: Extended Data Table 1. Examples of Rosetta simultaneous measurements. This dataset has been used for calculating the FUV atomic emission brightnesses at two times during FUV nadir-viewing observation periods (fourth and fifth cases in Figure 2): (1) the electron differential flux $J_{e}$ (see Extended Data Fig.1) measured by the RPC-IES electron spectrometer at the selected day and start time $t^{\mathrm{IES}}$ (first and second columns), at a cometocentric distance $r_{R}$ (third column), and associated with a number density $n_{e}^{\mathrm{IES}}$ (fourth column) and mean energy $E_{e}^{\mathrm{IES}}$ (fifth column) of electrons with energies between $10 \mathrm{eV}$ and $200 \mathrm{eV}$; (2) the total neutral density $n_{\text {tot }}^{\text {Cops }}$ measured by the ROSINA-COPS pressure gauge (sixth column) from which the column density $C^{\text {COPS }}$ is derived (seventh column); (3) the neutral composition measured by the ROSINA-DFMS neutral mass spectrometer and given in terms of volume mixing ratio $v_{n}$ of the four major neutral species (eighth column).

\begin{tabular}{|c|c|c|c|c|c|c|c|}
\hline Selected day & $\begin{array}{l}t^{\mathrm{IES}} \\
\text { (UT) }\end{array}$ & $\begin{array}{c}r_{R} \\
(\mathrm{~km})\end{array}$ & $\begin{array}{c}n_{e}^{\mathrm{IES}} \\
\left(\mathrm{cm}^{-3}\right)\end{array}$ & $\begin{array}{l}E_{e}^{\mathrm{IES}} \\
(\mathrm{eV})\end{array}$ & $\begin{array}{l}n_{t o t}^{\mathrm{COPS}} \\
\left(\mathrm{cm}^{-3}\right)\end{array}$ & $\begin{array}{c}C^{\mathrm{COPS}} \\
\left(10^{15} \mathrm{~cm}^{-2}\right)\end{array}$ & $\begin{array}{c}v_{n}^{\text {DFMS }} \\
\mathrm{H}_{2} \mathrm{O}, \mathrm{CO}_{2}, \mathrm{CO}, \mathrm{O}_{2}(\%)\end{array}$ \\
\hline 29 Mar 2015 & 11:47:18 & 92 & $30^{a}$ & $31^{a}$ & $12.5 \times 10^{6}$ & $6.2^{b}$ & $96,1.4,1.6,1.0^{c}$ \\
\hline 26 Dec 2015 & 08:35:08 & 79 & $1^{a}$ & $20^{a}$ & $13.5 \times 10^{6}$ & $4.9^{b}$ & $95,2.5,1.1,1.4^{c}$ \\
\hline
\end{tabular}

${ }^{a}$ The number density $n_{e}$ (fourth column) and mean energy (fifth column) of electrons with energies between $10 \mathrm{eV}$ and $200 \mathrm{eV}$ are derived by integrating the differential electron flux $J_{e}$ (corrected for the spacecraft potential) over the velocity space. These quantities are given for information; only $J_{e}$, not its moments, is used in the calculation of the modelled FUV brightnesses. ${ }^{b}$ The total column density is derived from the total neutral density $n_{\text {tot }}$ assuming a mean cometocentric distance for the nucleus' surface of $1.7 \mathrm{~km}^{48}$ (see Eq. 4). ${ }^{c}$ The volume mixing ratio for the four major neutral species is obtained from the ROSINA/DFMS mass spectrometer (other species are neglected). 
(a)

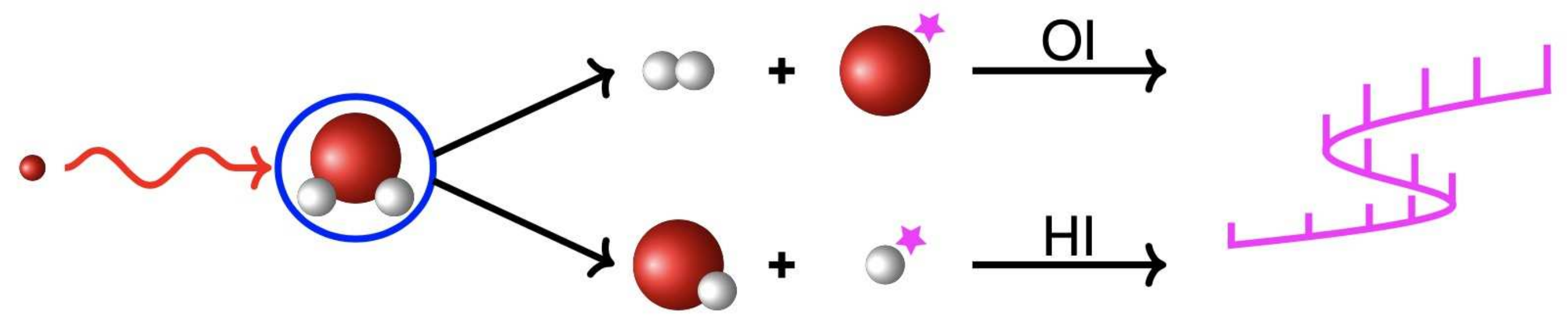

Energetic electron

RPC
Cometary molecule ROSINA
MIRO, VIRTIS
Excited atom
$\mathrm{HI}$ and $\mathrm{Ol}$ auroral emissions

Alice Modelled brightness

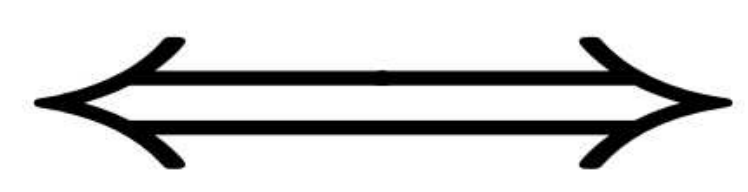

Observed brightness 

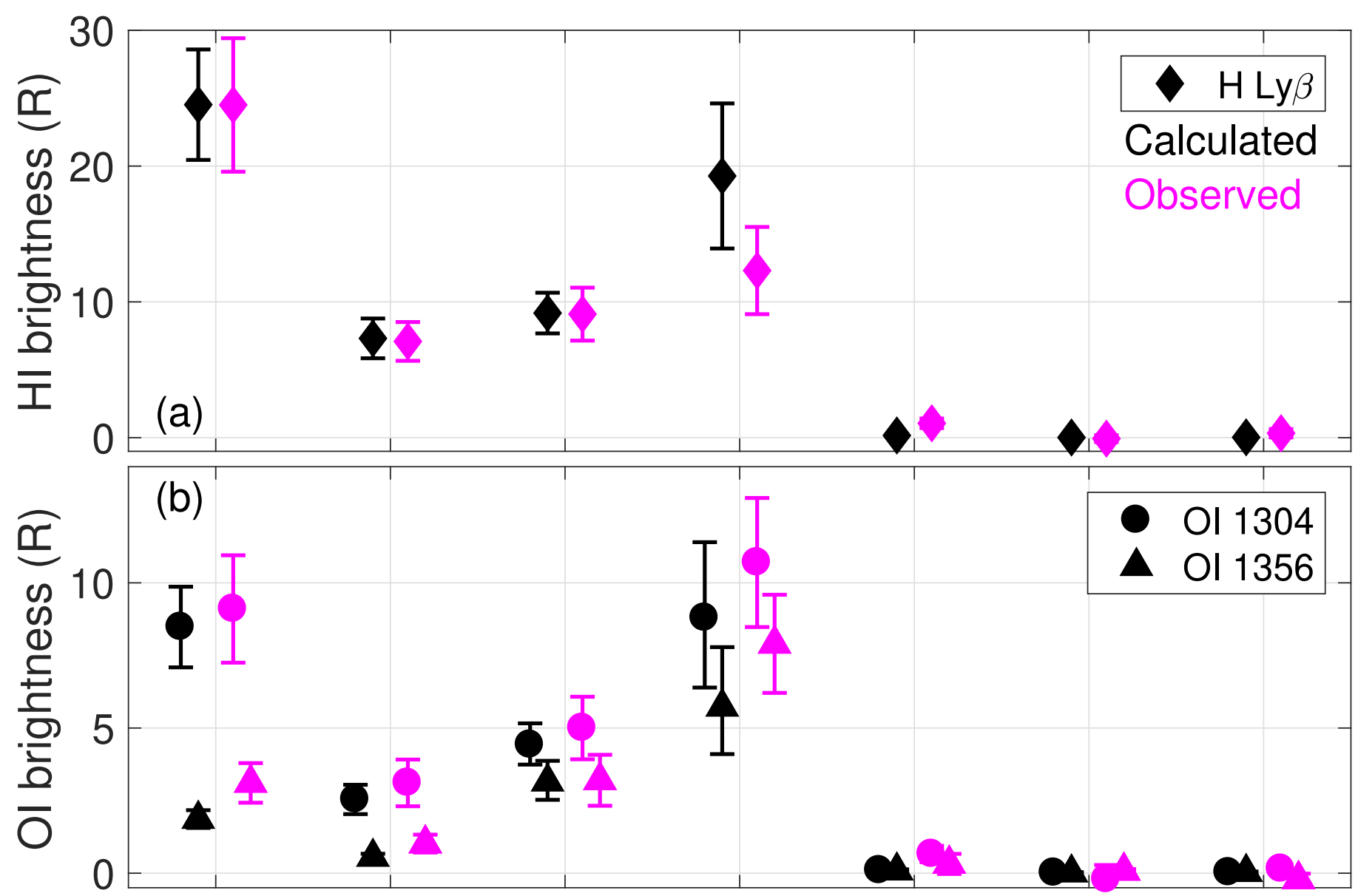

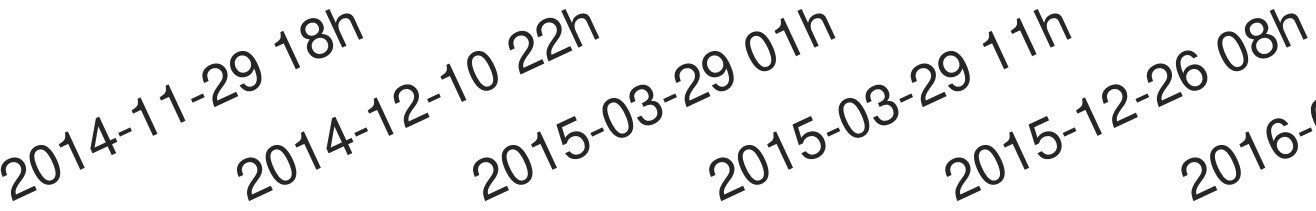



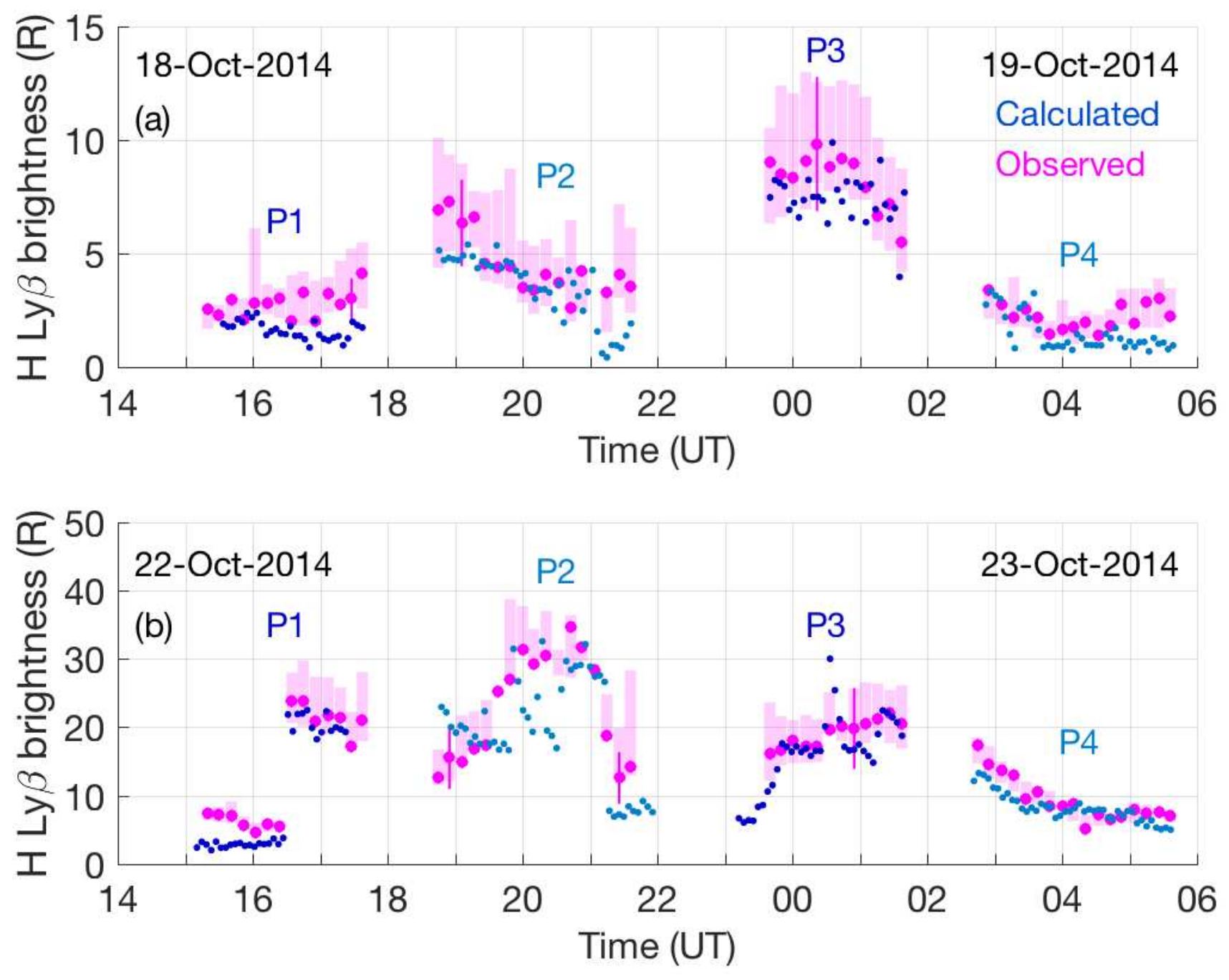


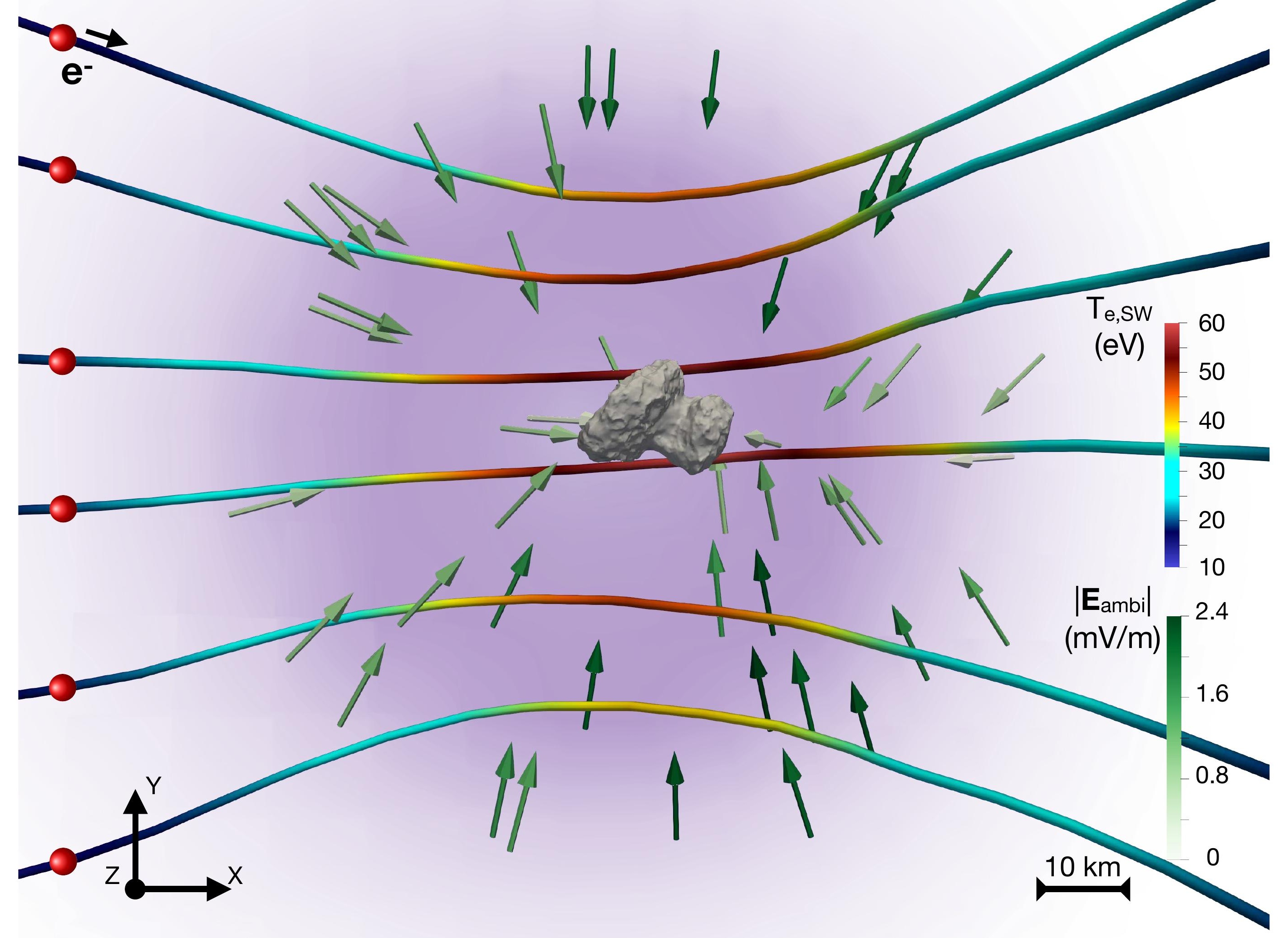




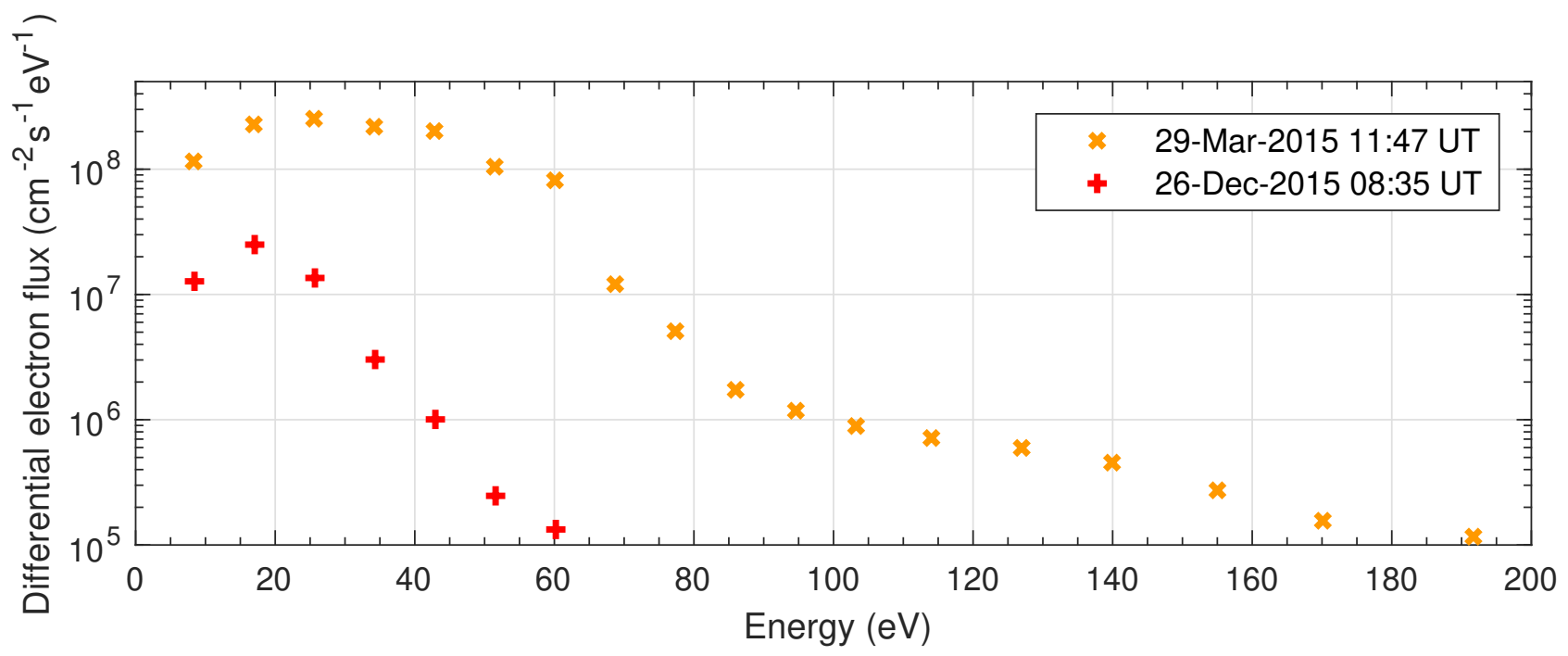

